\title{
Herschel Footprint Database and Service
}

\author{
E. Varga-Verebélyi ${ }^{1}$, L. Dobos ${ }^{2}$, T. Budavári ${ }^{3}$ and Cs. Kiss ${ }^{1}$ \\ ${ }^{1}$ Konkoly Observatory of the Hungarian Academy of Sciences, Budapest, Hungary \\ ${ }^{2}$ Department of Physics of Complex Systems, Eötvös Loránd University, Budapest, Hungary \\ ${ }^{3}$ Department of Applied Mathematics and Statistics, The Johns Hopkins University, \\ Baltimore, Maryland, USA
}

\begin{abstract}
We created the Herschel1 Footprint Database and web services for the Herschel Space Observatory imaging data. For this database we set up a unified data model for the PACS and SPIRE Herschel instruments, from the pointing and header information of each observation, generated and stored sky coverages (footprints) of the observations in their exact geometric form. With this tool we extend the capabilities of the Herschel Science Archive by providing an effective search tool that is able to find observations for selected sky locations (objects), or even in larger areas in the sky.
\end{abstract}

Keywords. astronomical data bases, infrared: general, space vehicles: instruments

\section{Introduction}

The Herschel Space Observatory (Pilbratt et al.2010) was a cornerstone mission of the European Space Agency, in operation between 2009 - 2013. The two imaging instruments, the PACS (Poglitsch et al.2010) and SPIRE (Griffin et al.2010) photometers covered the far-infrared and submillimeter wavelengths $(55-672 \mu \mathrm{m})$ and studied the formation of galaxies in the early Universe, their subsequent evolution and investigated the formation of stars and their interaction with the interstellar medium. All observations performed by Herschel are stored in the Herschel Science Archive (HSA) and accessible through the HSA User Interface2 for the astronomical community.

We extended the capabilities of the HSA by providing a tool that can search for measurements (OBSIDs) based on sky coordinates, identify the area covered by specific measurements (footprint) and provide capabilities for effective astronomical queries. The Herschel Footprint Database was built using the solutions applied in previous footprint database services like the Sloan Digital Sky Survey (Budavári et al., 2010). Our aim was to build a database and Herschel specific toolkit that is able to find each observation that included a selected sky position (or object) and its neighbourhood, or can identify observations that covered a certain area in the sky.

To prepare our own footprint database, it was necessary to obtain the information on the exact bore-sight in any type of observation, irrespectively of the scientific content of the measurement. We created a unified data model for the PACS and SPIRE Herschel instruments from their originally different data structures and downloaded the sky coverage information for each observation.

\section{Overview}

The unified data model was created using the pointing and header informations of all scan maps (PACS, SPIRE and PARALLEL mode observations) from the HSA. Scan maps in the HSA are usually processed at Level 2 or 2.5 which are the equivalent of flux-calibrated mosaic images in FITS format with a mask. Reconstructing the exact footprints of scan maps from these highly processed images would be challenging, mostly due to concave regions. Therefore,

1 Herschel is an ESA space observatory with science instruments provided by European-led Principal Investigator consortia and with important participation from NASA.

2 http://archives.esac.esa.int/hsa/ui/hui.jnlp 

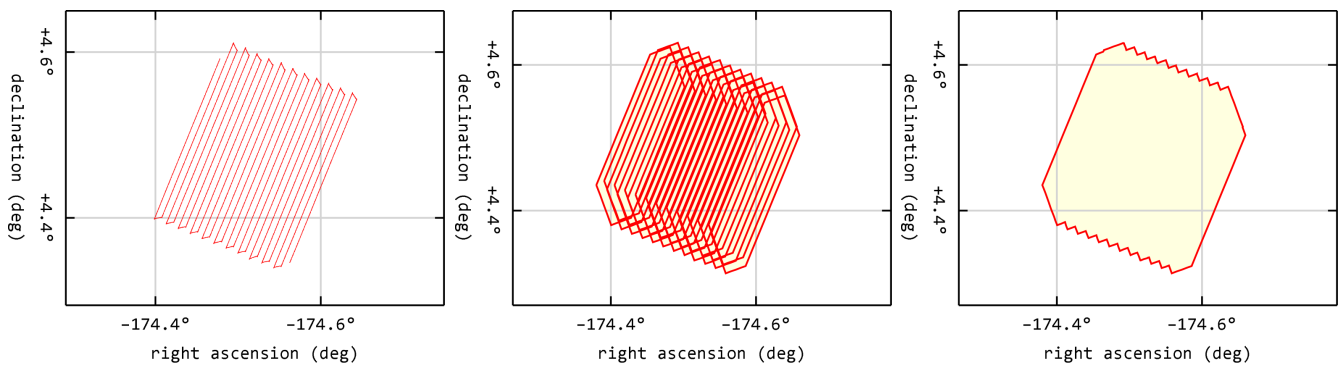

Figure 1. Reconstruction of the footprint from raw pointing data of PACS. Left panel: Raw pointing data, with telescope turn-arounds. These turn-arounds are filtered out and the entire scan curve is split into individual scan legs. Middle panel: Footprints of the individual, overlapping scan legs. Right panel: Final footprint, generated by taking the union of individual scan legs. Calculating the exact union by removing overlapping regions is necessary to determine the area of the footprint.

we had to reach back to initial pointing data - the pointing history of the telescope in a certain time window - and calculate footprints from the trajectories swept by the field of view of the various instruments.

Sky coverage is stored in exact geometric form allowing for precise area calculations of scan legs and the entire map. To describe celestial regions in an analytic form we upgraded the Spherical Library by Budavári et al.(2010) designed with astronomical use cases in mind. From the subsequent processing of the raw pointing information the generated footprint database was entirely implemented in SQL. Figure 1. shows an example and explanation of the reconstruction of a footprint from raw pointing data.

For a powerful and useful database the proper indexing is essential. Orders of magnitude (or more) faster search can be done when we properly arrange indexing and linking our different kind of data. We used the HTM indices Kunszt et al.(2000) for the Herschel Footprint Database. We also created a visualization tool to display footprints with two type of methods to reduce the complexity of outlines.

With this database users can easily inspect if an area or a specific position in the sky was observed by the Herschel or not. The database calculate the exact area of the observations and visualise it. The database is accessible via a web site (http://herschel.vo.elte.hu) and also as a set of REST web service functions which makes it usable from program clients like Python or IDL scripts. Data will be available in various formats including Virtual Observatory standards.

\section{References}

Budavári, T., Szalay, A. S., Fekete, G. 2010, PASP, Vol.122, p.1375

Griffin, M.J., Abergel, A., Abreu, A. et al. 2010, A\&A, 518, L3

Kunszt, P.Z., Szalay, A.S., Csabai, I., Thakar, A. R. 2000, ASPCS, Vol.216, p.141

Pilbratt, G.L., Riedinger, J.R., Passvogel, T. et al. 2010, A\&A, 518, L1

Poglitsch, A., Waelkens, C., Geis, N. et al. 2010, A\&A, 518, L2

\section{Acknowledgement}

The research was supported by the Hungarian OTKA NN 114560 grants and PECS Contract Nr. 4000109997/13/NL/KML of the Hungarian Space Office and the ESA. 